\title{
MONITORAMENTO DAS CARACTERÍSTICAS FÍSICO- QUÍMICAS E DE TEXTURA DE PRODUTO RESULTANTE DA INTERESTERIFICAÇÃO ENZIMÁTICA DE GORDURA DO LEITE COM ÓLEO DE CASTANHA-DO-PARÁ
}

\author{
G. F. M. NUNES ${ }^{1}$; A. V. de PAULA ${ }^{2}$; H. F. de CASTRO ${ }^{1}$; J. C. dos $\operatorname{SANTOS}^{3}$ \\ ${ }^{1}$ Universidade de São Paulo (USP), Escola de Engenharia de Lorena (EEL), Departamento de \\ Engenharia Química \\ ${ }^{2}$ Universidade Estadual Paulista(UNESP), Faculdade de Ciências Farmacêuticas, Departamento de \\ Engenharia Química \\ ${ }^{3}$ Universidade de São Paulo (USP), Escola de Engenharia de Lorena (EEL) ,Departamento de \\ Biotecnologia
}

E-mail para contato: jsant200@yahoo.com.br

\begin{abstract}
RESUMO - A modificação de lipídeos por interesterificação possibilita a obtenção de produtos alimentícios com características específicas e desejáveis, seja do ponto de vista nutricional, seja quanto à aceitabilidade pelo consumidor. Neste trabalho, a blenda de gordura do leite e óleo de castanha-do-pará, contendo 50\% deste, foi interesterificada em reação catalisada pela lipase de Rhizopus oryzae imobilizada em sílica-álcool polivinílico, a $45^{\circ} \mathrm{C}$. A reação foi avaliada pela composição do produto em triacilgliceróis, prevista através de um programa computacional, e monitorada pela consistência obtida em texturômetro e pelo conteúdo de gordura sólida (CGS), obtido por calorimetria de varredura diferencial (DSC). Os resultados mostraram que a consistência e o CGS, ambos medidos a $10^{\circ} \mathrm{C}$, podem ser correlacionados de forma linear, permitindo prever que produtos que apresentarem valores de CGS acima de 30\%, terão consistência dentro da faixa de $200-800 \mathrm{gf} / \mathrm{cm}^{2}$, com espalhabilidade satisfatória para "spreads".
\end{abstract}

\section{INTRODUÇÃO}

A gordura do leite é uma das principais fontes de lipídeos na alimentação humana, porém seu consumo é limitado devido à sua baixa espalhabilidade na temperatura de refrigeração, bem como por seu elevado conteúdo de ácidos graxos saturados, os quais frequentemente são relacionados à promoção de doenças coronárias (Lubary et al., 2011).

Para sanar este problema, processos que conduzam à obtenção de produtos a base de gordura do leite que sejam mais saudáveis e que tenham boa espalhabilidade à temperatura de refrigeração têm sido desenvolvidos. Dentre as tecnologias em desenvolvimento, destaca-se o processo de interesterificação enzimática, o qual representa uma alternativa interessante frente à hidrogenação 


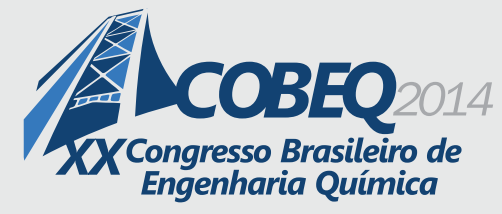

parcial de gorduras, técnica utilizada para produção de margarinas, que resulta na formação das chamadas gorduras trans, cuja ingestão relaciona-se a promoção de uma série de doenças, sendo consideradas mais prejudiciais à saúde que as gorduras saturadas presentes na manteiga (Nunes et al., 2010) .

Em meio aos óleos para interesterificação com a gordura do leite, o óleo de castanha-do-pará é uma opção interessante devido ao seu elevado valor nutricional, apresentando em sua composição principalmente os ácidos graxos oléico (C 18:1) (monoinsaturado) e linoléico (C 18:2) (essencial) (Bentes-Gama et al., 2007), além de conter um aroma bastante agradável (Campestre, 2011).

Neste contexto, o objetivo deste trabalho foi estudar as características físico-químicas e de textura de produto resultante da interesterificação enzimática de gordura do leite com óleo de castanha-do-pará, visando à obtenção de um alimento mais saudável e com melhor espalhabilidade à temperatura de refrigeração em comparação à manteiga. Para tal finalidade, blendas de gordura do leite e óleo de castanha-do-pará foram interesterificadas utilizando a lipase de Rhizopus oryzae imobilizada sílica-álcool polivinílico $\left(\mathrm{SiO}_{2}\right.$-PVA) em um sistema livre de solvente.

\section{MATERIAIS E MÉTODOS}

Como biocatalisador, empregou-se lipase de Rhizopus oryzae (L036P, Biocatalysts, Cardiff, England), adquirida em sua forma livre e posteriormente imobilizada por adsorção física em suporte híbrido polixilossano-álcool polivinílico $\left(\mathrm{SiO}_{2}-\mathrm{PVA}\right)$, conforme metodologia descrita por Nunes (2011). A gordura do leite foi obtida a partir de fusão completa de manteiga comercial (Aviação sem sal, adquirida em mercado local) seguida de centrifugação (2136g/10min) e separação da fase aquosa. O óleo de castanha-do-pará adquirido da Inovam Brasil (Ji-Paraná/RO) foi utilizado sem nenhum tratamento adicional.

Os experimentos foram efetuados em reatores cilíndricos encamisados, carregados com $40 \mathrm{~g}$ de meio, na proporção mássica de 50\% de gordura do leite e 50\% de óleo de castanha-do-pará, e incubados com 500 unidades de atividade de lipase imobilizada por grama de meio. Foram incubados 6 reatores sob agitação magnética (150rpm), em atmosfera inerte de nitrogênio, sob o abrigo da luz, à temperatura de $45^{\circ} \mathrm{C}$, por um período máximo de $48 \mathrm{~h}$. A composição em triacilgliceróis (TAGs) quanto ao seu grau de saturação foi prevista a partir do programa computacional 1,2,3 óleos proposto por Antoniosi Filho (1995) e considerando-se o uso de lipase $s n-1,3$ específica. O acompanhamento da consistência dos produtos $\left(10^{\circ} \mathrm{C}\right)$ foi realizado em analisador de textura (QTS-25 Brookfield) e o conteúdo de gordura sólido (CGS) foi calculado a partir dos dados obtidos em análises térmicas realizadas em Calorímetro de Varredura Diferencial DSC (SII Nanotechnology - Seiko, Modelo 6220; Northridge, U.S. A). Todas as análises e cálculos foram realizados conforme metodologia descrita por Nunes (2011).

\section{RESULTADOS E DISCUSSÃO}

A Tabela 1 apresenta a composição da gordura do leite, óleo de castanha-do-pará e blenda (50:50) gordura do leite:óleo de castanha-do-pará, antes e após a interesterificação enzimática 
catalisada por lipase sn-1,3 específica, segundo teores de TAGs dos tipos trissaturados (SSS), dissaturado-monoinsaturados (SSI), di-insaturados-monossaturado (IIS) e tri-insaturados (III).

Tabela 1 - Composição em triacilgliceróis (TAGs) segundo grau de saturação para as blendas de gordura do leite e óleo de castanha-do-pará antes e após a interesterificação enzimática catalisada por lipase $s n-1,3$ específica

\begin{tabular}{|c|c|c|c|c|c|c|}
\hline \multicolumn{2}{|r|}{ Tipo de TAG } & SSS (\%) & SSI (\%) & IIS (\%) & III $(\%)$ & Total $(\%)$ \\
\hline blenda & NIE & 17,98 & 41,27 & 15,98 & 24,78 & 100,00 \\
\hline $50: 50 *$ & EIE & 10,46 & 37,52 & 37,26 & 14,76 & 100,00 \\
\hline
\end{tabular}

*Proporção mássica gordura do leite: óleo de castanha-do-pará; S = Saturado; I = Insaturado. $\mathrm{NIE}=$ não interesterificado; $\mathrm{EIE}=$ enzimaticamente interesterificado.

A Figura 1 apresenta a consistência e a Figura 2a mostra o CGS em função do tempo de reação para os produtos obtidos na interesterificação, sendo que a Figura $2 b$ mostra a relação entre os valores de CGS e consistência, ambos a $10^{\circ} \mathrm{C}$, para os produtos interesterificados nos diferentes tempos reacionais.

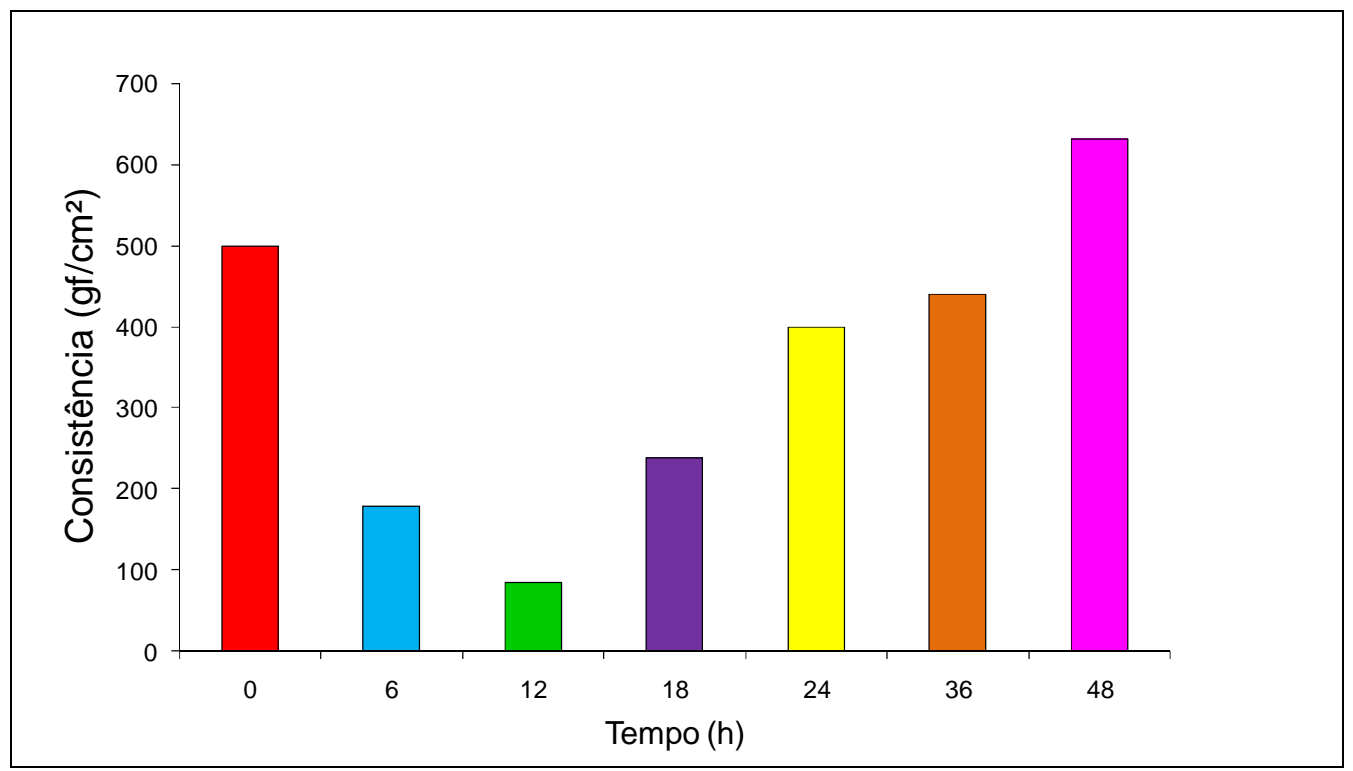

Figura 1 - Consistência em função do tempo de reação para o produto obtido na interesterificação enzimática da blenda contendo proporção mássica de 50\% de gordura do leite e $50 \%$ óleo de castanha do pará

A Figura 1 indica uma variação acentuada de consistência até cerca de $600 \mathrm{gf} / \mathrm{cm}^{2}$. Verifica-se ainda uma tendência inicial de redução da consistência nas primeiras $12 \mathrm{~h}$ de reação, seguida de um comportamento inverso alcançando cerca de $650 \mathrm{gf} / \mathrm{cm}^{2}$ ao final da reação. 


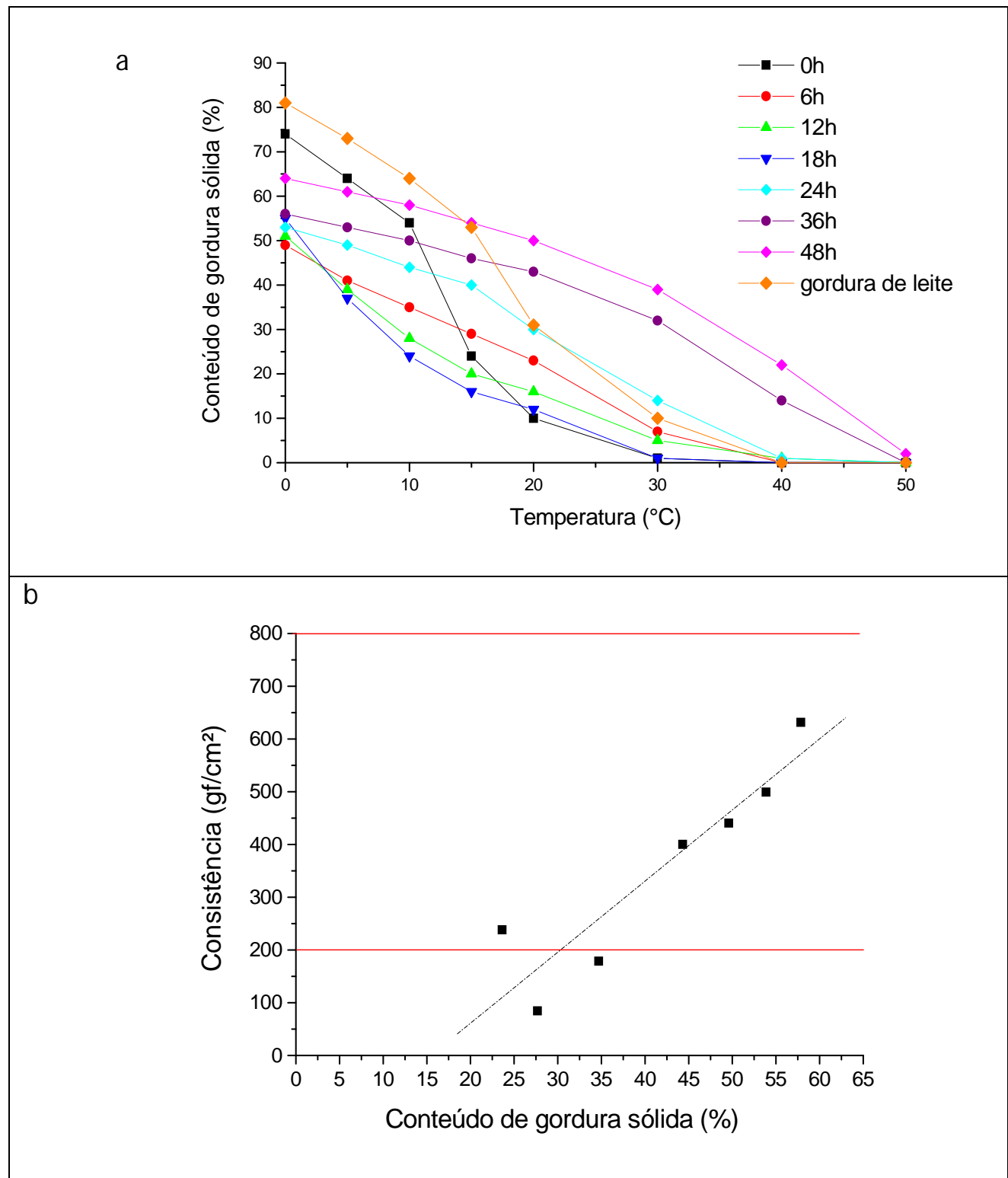

Figura 2 - (a) Conteúdo de gordura sólida (CGS) em função do tempo de reação para o produto obtido na interesterificação enzimática. (b) Relação entre CGS e consistência, ambos a $10^{\circ} \mathrm{C}$, para os produtos interesterificados da blenda contendo proporção mássica de $50 \%$ de gordura do leite e $50 \%$ óleo de castanha-do-pará nos diferentes tempos reacionais

Apenas os produtos obtidos a partir de $18 \mathrm{~h}$ de reação apresentaram valores de consistência dentro da faixa estabelecida por Haighton (1959) $\left(200\right.$ a $\left.800 \mathrm{gf} / \mathrm{cm}^{2}\right)$ para uma gordura com satisfatória espalhabilidade. 


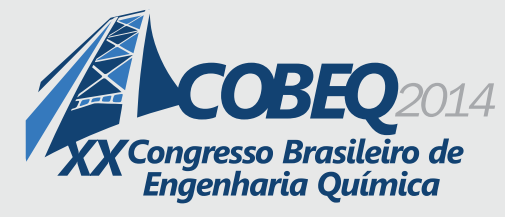

19 a 22 de outubro de 2014

Florianópolis/SC

Comparando-se os conteúdos de gordura sólida da gordura do leite aos da blenda não interesterificada, pode-se observar que o acréscimo do óleo de castanha-do-pará à gordura do leite resultou na redução deste parâmetro para todas as temperaturas de análise. Ainda assim, a blenda não interesterificada apresentou elevado teor de sólidos a temperatura de $10^{\circ} \mathrm{C}(54 \%)$, o que pode ser correlacionada à presença de grande quantidade de TAGs SSS+SSI (59\%, Tabela $1)$.

Após a reação, observou-se que, a $10^{\circ} \mathrm{C}$, dentre os produtos obtidos, o de $48 \mathrm{~h}$ apresentou conteúdo de gordura sólida acima do valor de sólidos da blenda não interesterificada (NIE) (0 h). Estes resultados estão em acordo com os resultados de consistência, já que dentre os produtos obtidos ao longo tempo, apenas o produto de $48 \mathrm{~h}$ de processo apresentou consistência superior a blenda NIE (Figura 1).

Pelos valores de conteúdo de gordura sólida, apresentados na Figura 2a, observa-se que, dentre os produtos obtidos ao longo do tempo, segundo os critérios apresentados por O'Brien (2004), os que apresentaram melhor plasticidade foram os obtidos com 12 e 18 h de reação, em função dos teores de sólido ideais para um "spread" a $10^{\circ} \mathrm{C}$ (próximos de $30 \%$ ), boa resistência a migração do óleo a $20^{\circ} \mathrm{C}$ (sólidos > 10\%) e teor de sólidos próximos a zero a $30^{\circ} \mathrm{C}$ (sólidos < $3 \%$ ), mostrando serem, portanto adequados para aplicações nesta temperatura, já que neste caso não devem apresentar arenosidade na boca. Porém, o produto obtido em $12 \mathrm{~h}$ reação apresentou consistência muito baixa a $10^{\circ} \mathrm{C}\left(<200 \mathrm{gf} / \mathrm{cm}^{2}\right)$. Para o produto obtido em $18 \mathrm{~h}$, quando mantido em temperatura ambiente $\left(\sim 25^{\circ} \mathrm{C}\right)$ foi visualmente perceptível a migração do óleo, além de ter apresentado consistência a $10^{\circ} \mathrm{C}$ muito próxima do limite inferior da faixa determinada para um "spread" com boa espalhabilidade $\left(\sim 200 \mathrm{gf} / \mathrm{cm}^{2}\right)$.

Neste sentido, o produto que apresentou consistência dentro da faixa indicada com boa espalhabilidade (entre 200 a $800 \mathrm{gf} / \mathrm{cm}^{2}$ ), a $10^{\circ} \mathrm{C}$, e que não apresentou visualmente migração de óleo quando mantido em temperatura ambiente $\left(\sim 25^{\circ} \mathrm{C}\right)$, foi o obtido em $24 \mathrm{~h}$ de reação (Figura 1), apesar de ter apresentado conteúdo de gordura sólida, segundo os critérios de O'Brien (2004) elevado a $10^{\circ} \mathrm{C}(44 \%)$. Isso pode ser explicado pelo fato de que o conteúdo de gordura sólida não é a única variável que influencia a consistência de uma gordura, pois, além da relação entre a quantidade de sólido e líquido presente na gordura, o caráter cristalino da fase sólida presente também é uma variável de fundamental importância na determinação da consistência do produto (Ribeiro et al. 2009).

Verifica-se que a curva de conteúdo de gordura sólida para os produtos obtidos a partir de $24 \mathrm{~h}$, não apresenta uma inclinação tão acentuada quanto a curva da mistura $(0 \mathrm{~h})$, e esse comportamento pode ampliar a faixa de fusão do produto, possibilitando o aumento do conteúdo de gordura sólida em temperaturas mais elevadas, como a $20^{\circ} \mathrm{C}$, favorecendo outras propriedades além da consistência, como a menor exsudação do óleo, por exemplo. Cabe ainda observar que, embora a consistência do produto obtido em $24 \mathrm{~h}$ seja semelhante à da mistura, o valor de sólidos $\left(\mathrm{a} 10^{\circ} \mathrm{C}\right)$ deste produto $(44 \%)$ apesar de considerado elevado segundo os critérios de O'Brien (2004), é $10 \%$ mais baixo que valor de sólidos da mistura (0 h) (54\%), o que sugere que a reação também resultou em redução desta propriedade.

Como a consistência de gorduras é influenciada por seu CGS, foi estabelecida uma relação entre estas duas propriedades físicas, ambas determinadas a $10^{\circ} \mathrm{C}$, para os produtos das reações de interesterificação (Figura 2b). A partir da linearização dos dados experimentais 
dispostos nesta figura, foi possível obter a Equação (1), com $\mathrm{R}^{2}=0,9511$, em que CGS = conteúdo de gordura sólida $(\%)$.

$$
\text { Consistência }\left(\mathrm{gf} / \mathrm{cm}^{2}\right)=-208,52+13,48 . \mathrm{CGS}
$$

De acordo com esta equação, produtos que apresentem valores de conteúdo de gordura sólida acima de $30 \%$ proporcionarão consistência dentro da faixa de 200-800 gf $/ \mathrm{cm}^{2}$, estabelecida por Haighton (1959) com espalhabilidade satisfatória para "spreads".

\section{CONCLUSÃO}

Os resultados mostraram que há uma relação entre a composição química em triacilgliceróis e as propriedades físicas (consistência e conteúdo de gordura sólida) do produto alimentício obtido. Além disso, observou-se que os valores de consistência e conteúdo de gordura sólida podem ser correlacionados de forma linear, o que permite predizer que produtos obtidos a partir das condições experimentais estudadas, que apresentarem dados de conteúdo de gordura sólida acima de $30 \%$, proporcionarão consistência dentro da faixa de $200-800 \mathrm{gf} / \mathrm{cm}^{2}$, com espalhabilidade satisfatória para "spreads".

\section{NOMENCLATURA}

CGS = Conteúdo de gordura sólida

$\mathrm{DSC}=$ Calorimetria de varredura diferencial

$\mathrm{EIE}=$ Enzimaticamente interesterificado

$\mathrm{I}=$ Insaturado.

NIE = não interesterificado;

$\mathrm{S}=$ Saturado;

TAGs $=$ triacilgliceróis

\section{AGRADECIMENTOS}

Os autores agradecem a FAPESP, a CAPES e ao CNPq pelo apoio financeiro concedido para o desenvolvimento deste trabalho.

\section{REFERÊNCIAS}

BENTES-GAMA, M. M.; VIEIRA, A. H.; LIMA, L. F.; DE OLIVEIRA, A. C.; DA SILVA, A. P. F. F. Ocorrência de populações naturais de espécies não-madeireiras em Rondônia. Empresa Brasileira de Pesquisa Agropecuária - EMBRAPA, 2007. 
CAMPESTRE IND. E COM. DE ÓLEOS VEGETAIS LTDA. Óleo de Castanha. Disponível em: http://www.campestre.com.br/oleo-de-castanha-para.shtml/ Acessado em: 19 de agosto de 2011.

LUBARY, M.; HOFLAND, G. W.; TER HORST, J. H. The potential of milk fat for the synthesis of valuable derivatives. European Food Research and Technology. v. 232, p. 1-8, 2011.

NUNES, G. F. M.; PAULA, A. V.; DE CASTRO, H. F.; SANTOS, J. C. Modificação bioquímica da gordura do leite. Química Nova, v. 33, n. 2, p. 431-437, 2010.

RIBEIRO, A.P. B.; BASSO, R. C.; GRIMALDI, R.; GIOIELLI, L. A.; GONÇALVES, L. A. G. Instrumental methods for the evaluation of interesterified fats. Food Analytical Methods, 2009, doi: 10.1007/s12161-009-9073-4.

ANTONIOSI FILHO, N. R. Análise de óleos e gorduras vegetais por meio de métodos cromatográficos de alta resolução e métodos computacionais. 1995. Tese (Doutorado em Química Analítica) - Universidade de São Paulo, São Carlos/SP.

HAIGHTON, A. J. The measurement of the hardness of margarine and fats with cone penetrometers. J. Am. Oil Chem. Soc., v. 36, n. 8, p. 345-348, 1959.

NUNES, G. F. M. Interesterificação enzimática de misturas binárias e ternárias de gordura do leite com óleos de castanha-do-pará e canola: Avaliação de estratégias de maximização do desempenho do processo e das propriedades texturais e sensoriais do produto. 2011. Tese (Doutorado em Biotecnologia Industrial). Escola de Engenharia de Lorena; Universidade de São Paulo, Lorena/SP.

O'BRIEN, R. D. Fats and Oils: Formulating and Processing for Applications. 2nd ed. New York: CRC Press LLC, 2004. 